\title{
Toward structurally integrated locally resonant metamaterials for vibration attenuation
}

Jascha U. Schmied, Christopher Sugino, Andrea Bergamini, Paolo Ermanni, Massimo Ruzzene, et al.

Jascha U. Schmied, Christopher Sugino, Andrea Bergamini, Paolo Ermanni, Massimo Ruzzene, Alper Erturk, "Toward structurally integrated locally resonant metamaterials for vibration attenuation," Proc. SPIE 10164, Active and Passive Smart Structures and Integrated Systems 2017, 1016413 (11 April 2017); doi: 10.1117/12.2260306 Health Monitoring, 2017, Portland, Oregon, United States 


\title{
Toward structurally-integrated locally resonant metamaterials for vibration attenuation
}

\author{
Jascha U. Schmied ${ }^{\mathrm{a}}$, Christopher Sugino ${ }^{\mathrm{b}}$, Andrea Bergamini ${ }^{\mathrm{c}}$, Paolo Ermanni ${ }^{\mathrm{a}}$, Massimo \\ Ruzzene $^{\mathrm{b}}$, and Alper Erturk ${ }^{\mathrm{b}}$ \\ ${ }^{a}$ ETH Zurich, Leonhardstrasse 21, Zürich, Switzerland \\ ${ }^{\mathrm{b}}$ Georgia Institute of Technology, 771 Ferst Drive, Atlanta, Georgia, United States \\ ${ }^{\circ}$ Empa, Überlandstrasse 129, Dübendorf, Switzerland
}

\begin{abstract}
In this contribution, we explore the use of locally resonant metamaterials for multi-functional structural loadbearing concepts using analytical, numerical, and experimental techniques. Locally resonant metamaterials exhibit bandgaps at wavelengths much larger than the lattice dimension. This is a promising feature for lowfrequency vibration attenuation. The presented work aims to investigate highly integrated structural concepts and experimentally validated prototypes for vibration reduction in load-bearing applications. The goal is to explore and extend the design space of lightweight structural systems, by designing multi-functional periodic structural elements, preserving structural stiffness while concurrently enabling sufficiently wideband damping performance over a target frequency range of interest. Following a generalized theoretical modeling framework for bandgap design and analysis in finite structures, the focus is placed on the design, fabrication, and analysis of a load-carrying frame development with internally resonant components. Finite-element modeling is employed to design and analyze the frequency response of the frame and simplified analytical solution is compared with this numerical solution. Experimental validations are presented for a 3D-printed prototype. The effects of various parameters are reported both based on numerical and experimental findings.
\end{abstract}

Keywords: Metamaterial, Resonant, Vibration Attenuation, Numerical, Experimental, Analytical, Bandgap

\section{INTRODUCTION}

Low frequency broad band vibration attenuation is a topic crucial to lightweight structural engineering in aerospace applications among others. In such load bearing structures, excessive deformations have to be avoided to ensure lifetime safety, furthermore, sensitive sensor and delicate payloads have to be insulated from vibrations occuring through propulsion and aerodynamic effects of the carrier vehicle. At the same time, these structures are multifunctional, they have to carry static loads and be lightweight in order to minimize the total weight of the carrier vehicle and in turn reduce costs and fuel consumption. As a result, lightweight load bearing concepts for structures with low frequency broad band vibration attenuation have to be devised.

A metamaterial approach is chosen in order to achieve not only low frequency, but also broad band vibration attenuation. Using a single tuned mass damper allows only for narrow band vibration attenuation of a single resonance frequency of a structure. However, using an array of unit cell each containing a resonators allows for broad band vibration attenuation as long as the resonators are coupled through the structure. As a result of appropriate coupling the modes degenerate and create a broad band frequency attenuation phenomenon.

While in the previous work within this project the focus had been on high frequency vibration attenuation with a high amount of added mass which lead to a multiplication of the initial mass of the structure, the focus of the presented work has been on using stiff structures and adding relatively low amounts of mass in order to achieve feasibility for lightweight structural applications with non-lumped mass resonators. This was achieved in three steps, first load bearing structures with internal resonators were modelled with simplified finite element models

Further author information: (Send correspondence to Alper Erturk)

Jascha U. Schmied: E-mail: jschmied@ethz.ch, Telephone: +41 (0)44633 8740

Alper Erturk: E-mail: alper.erturk@me.gatech.edu, Telephone: +1 404-385-1394

Active and Passive Smart Structures and Integrated Systems 2017, edited by Gyuhae Park, Alper Erturk, Jae-Hung Han, Proc. of SPIE Vol. 10164, 1016413

(C) 2017 SPIE $\cdot$ CCC code: $0277-786 X / 17 / \$ 18 \cdot$ doi: $10.1117 / 12.2260306$ 
reducing the resonators to ideal mass spring systems. In a second step, a configuration was selected and designed in a way that it was manufacturable with 3D printing. Lastly, with the help of full 3D finite element models and experimental measurements with varying amounts of mass, the theoretical predictions where validated and numerical and experimental results where compared. It is worth mentioning that the designed prototype also included an extension of the resonators allowing to use inertial amplification in order to reduce the necessary mass in the resonators to address a given target frequency.

\section{THEORETICAL CONSIDERATION}

The general governing equations for locally resonant metastructure (e.g. rods, beams, membranes, plates) can be written as

$$
\begin{gathered}
\mathcal{L}[w(\mathbf{P}, t)]+m(\mathbf{P}) \ddot{w}(\mathbf{P}, t)-\sum_{j=1}^{S} k_{j} u_{j}(t) \delta\left(\mathbf{P}-\mathbf{P}_{j}\right)=f(\mathbf{P}, t), \quad \mathbf{P} \in D . \\
m_{j} \ddot{u}_{j}+k_{j} u_{j}+m_{j} \frac{\partial^{2} w}{\partial t^{2}}\left(\mathbf{P}_{j}, t\right)=0, \quad j=1 \ldots S
\end{gathered}
$$

where $\mathcal{L}$ is the linear, self-adjoint stiffness operator of the system, $m(\mathbf{P})$ is the mass distribution of the system, $w(\mathbf{P}, t)$ is the displacement of the structure at a point $\mathbf{P}$ and time $t, \delta(\mathbf{P})$ is the Dirac delta function, $k_{j}, m_{j}$, $\mathbf{P}_{j}$, and $u_{j}$, are the stiffness, mass, attachment point, and displacement of the $j$ th resonator respectively, and $S$ is the total number of resonators. The domain $D$ can be one- or two-dimensional, depending on the problem (e.g. one-dimensional for a beam, two-dimensional for a plate).

To discretize the partial differential equation, a series form of $w$ is assumed using the mode shapes of the plain structure (i.e. the structure without any resonators) as basis functions

$$
w(\mathbf{P}, t)=\sum_{r=1}^{N} \phi_{r}(\mathbf{P}) \eta_{r}(t)
$$

where $N$ is the total number of modes used in the expansion, and $\phi_{r}$ is the $r$ th mode shape of the plain structure, and $\eta_{r}$ is the $r$ th modal coordinate. It can be shown that the mode shapes $\phi_{r}$ can be normalized to satisfy the orthogonality conditions

$$
\begin{gathered}
\int_{D} m(\mathbf{P}) \phi_{i}(\mathbf{P}) \phi_{j}(\mathbf{P}) d D=\delta_{i j} \\
\int_{D} \mathcal{L}\left[\phi_{i}(\mathbf{P})\right] \phi_{j}(\mathbf{P}) d D=\omega_{i}^{2} \delta_{i j}
\end{gathered}
$$

where $\omega_{i}$ are the resonant frequencies of the plain structure, and $\delta_{i j}$ is the Kronecker delta. Substituting Eq. (3) into Eq. (1), multiplying by another mode shape, integrating across the domain, and applying the orthogonality conditions, the discretized set of ordinary differential equations for the modal coordinates are obtained as

$$
\ddot{\eta}_{r}(t)+\omega_{r}^{2} \eta_{r}(t)-\sum_{j=1}^{S} k_{j} u_{j}(t) \phi_{r}\left(\mathbf{P}_{j}\right)=q_{r}(t), \quad r=1,2, \ldots, N
$$

where

$$
q_{r}(t)=\int_{D} f(\mathbf{P}, t) \phi_{r}(\mathbf{P}) d D
$$

Taking the Laplace transforms of Eq. (2) and (6), the following system of linear algebraic equations can be obtained

$$
\left(s^{2}+\omega_{r}^{2}\right) H_{r}(s)+s^{2} \sum_{j=1}^{S} \frac{m_{j} \omega_{t}^{2}}{s^{2}+\omega_{t}^{2}} \phi_{r}\left(\mathbf{P}_{j}\right) \sum_{k=1}^{N} H_{k}(s) \phi_{k}\left(\mathbf{P}_{j}\right)=Q_{r}(s), \quad r=1,2, \ldots, N
$$


where $\omega_{t}=\sqrt{k_{j} / m_{j}}$ is the identical resonant frequency of every resonator, $s$ is the Laplace complex variable, and $H_{r}(s)$ and $Q_{r}(s)$ are the Laplace transforms of $\eta_{r}(t)$ and $q_{r}(t)$, respectively.

Assuming the structure has uniform mass distribution $m(\mathbf{P})$, the resonators have identical masses $m_{j}$, and that the resonators are placed uniformly on the structure, the following approximation can be used:

$$
\sum_{j=1}^{S} m_{j} \phi_{r}\left(\mathbf{P}_{j}\right) \phi_{s}\left(\mathbf{P}_{j}\right) \approx \mu \int_{D} m \phi_{r}(\mathbf{P}) \phi_{s}(\mathbf{P}) d D=\mu \delta_{r s}
$$

where

$$
\mu=\frac{1}{m_{\text {structure }}} \sum_{j=1}^{S} m_{j}=\frac{m_{\text {res }, \text { tot }}}{m_{\text {structure }}}
$$

where $m_{\text {structure }}$ is the total mass of the original structure and $m_{r e s, t o t}$ is the total mass of the resonators. With this approximation, the system of equations decouples, yielding

$$
H_{r}(s)=\frac{Q_{r}(s)}{s^{2}\left(1+\frac{\mu \omega_{t}^{2}}{s^{2}+\omega_{t}^{2}}\right)+\omega_{r}^{2}}
$$

With this simplification, it can be shown that no mode of the original structure can have resonant frequencies in the frequency range

$$
\omega_{t}<\omega<\omega_{t} \sqrt{1+\mu} .
$$

which defines the edge frequencies of the locally resonant bandgap. The width of the band gap $\Delta \omega$ is, as a result, proportional to the tuning frequency and furthermore depending on the added mass ratio $\mu$

$$
\Delta \omega=\omega_{t}(\sqrt{1+\mu}-1) .
$$

Sugino et al. have published and experimentally validated this hypothesis for the attenuation of high index modes $\omega_{i}$ with $i \geq 5$ and added mass ratio $\mu \geq 11^{1}{ }^{2}$ In this work, added mass ratios $\mu \leq 1.25$ are investigated.

\section{VALIDATION}

The relationship between added mass ratio $\mu$ to the attenuation region width has been validated experimentally by Sugino et al. ${ }^{1}$ The results of this investigation are presented in figure 1. Apart from deriving the analytical predictions, the beam was modeled using the governing equations in Eq. (2) and Eq. (6), which were solved numerically using matrix inversion for a cantilever beam excited by base motion. The bandgap edge frequencies were obtained using Eq. (12), and the mass ratio was calculated using Eq. (10), where the mass of the structure included the mass of the clamping hardware used to attach the resonators to the primary structure, and the mass of the resonators was assumed to be the sum of the tip masses attached to each resonators. The numerical and experimental investigations in this work were done for a bending beam with low bending stiffness, for the presented work the focus of the investigation is moved from such non-load bearing structures with attached resonators to load bearing structures with integrated multifunctional resonators.

\section{APPLICATION OF THE THEORY TO LOAD BEARING STRUCTURES}

The challenge of the application to load bearing lightweight structures lies in the clearly defined objectives and the resulting requirements. First and foremost, load bearing structures for aerospace applications have to be lightweight. As a result, the stiffness to mass ratio of the structures have to be high, furthermore, the added mass ratio has to be minimized. At the same time, vibration attenuation has to be realized at low frequencies, i.e. low index eigenmodes, and in broad band. However, achieving broad band vibration attenuation at low frequencies is typically associated with high weight penalties considering that the tuning frequency of the resonator is 


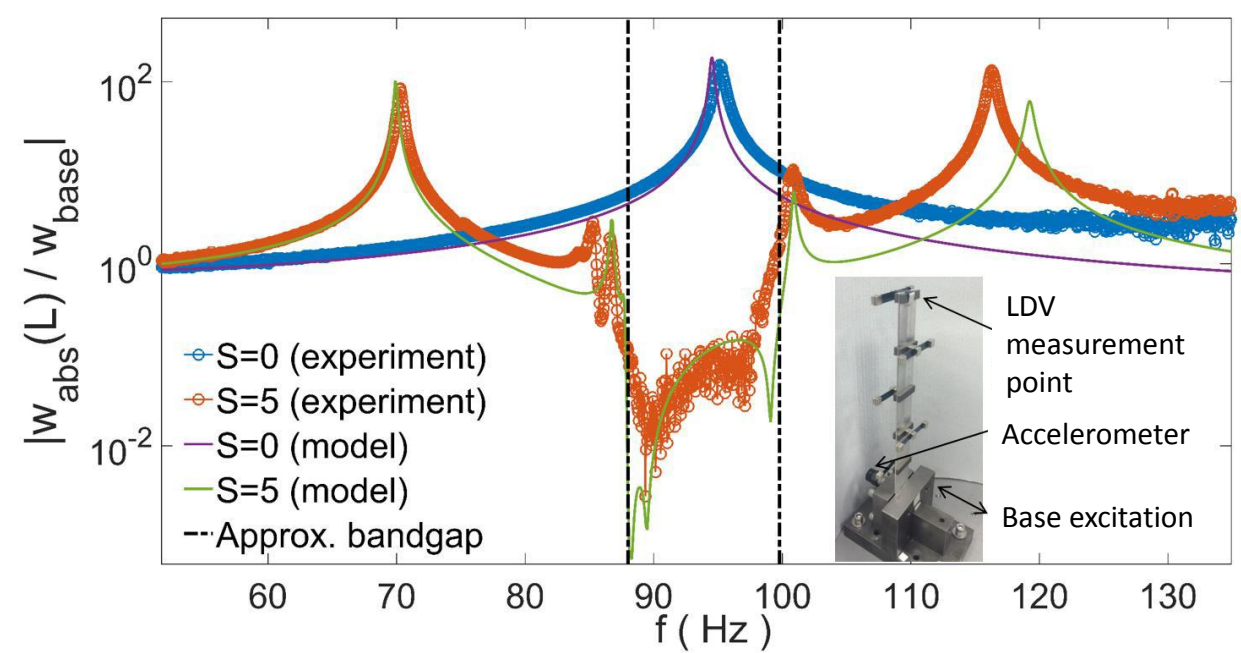

Figure 1. Comparison of experimental results and assumed modes model simulations. The bandgap approximation with an infinite number of absorbers $(S \rightarrow \infty)$ is also shown for reference. Insert shows the experimental setup of a vertical cantilever with five absorbers as a locally resonant metamaterial beam under base excitation. ${ }^{1}$

$\omega_{t}=\sqrt{\frac{k_{r e s}}{m_{\text {res }}}}$ and that equation 13 is valid for the width of the attenuation region $\Delta \omega$. Consequently, three approaches can be chosen.

Firstly, the stiffness of the resonators can be reduced, until they reach the limit of manufacturability and durability. As a result, the mass $m_{j}$ within each resonator is chosen appropriately to match the targeted eigenmode of the structure. The targeted eigenmode is preferably one of the lowest index eigenmodes $\omega_{i}$ mit $i \in\{1,2,3\}$. Furthermore, the stiffness of the structure is maximized to increase the lowest index eigenmodes.

Secondly, the resonators can be integrated into the structure so that they not only resonate, but also increase the stiffness of the structure significantly. As a result, the stiffness of the resonators $k_{r e s}$, as well as the stiffness of the structure and its eigenfrequencies $\omega_{i}$ are increased. Lastly, it is possible to use inertial amplification to reduce the mass of the resonator by exploiting the kinematics within the unit cell to increase the apparent inertia for a given mass within the resonator. The first and last approach are discussed in more detail in the subsequent sections of this contribution by presenting numerical and experimental results.

\subsection{Inertial Amplification}

Internally resonating structures and metamaterials have already seen multiple ways of implementation. However, they inherently show a direct relationship between the mass within the resonator and the stiffness of the spring with target frequency for vibration attenuation. A way to break this direct relationship was proposed by Yilmaz et al. In their work, they propose the introduction of kinematic coupling of different masses within a lattice in order to amplify the inertia of each point mass ${ }^{34} .^{5}$ They introduced the inertial amplification in a finite 2D lattice metamaterial to create phononic band gaps and compared them to the band gaps created by local resonance and Bragg scattering. In this work the concepts introduced by Yilmaz et al. are adapted to load bearing and vibrating structural components. In contrast to Yilmaz et al. the inertial amplification was not part in a quasi-homogenous material, but the resonators extended with inertial amplification were separated and coupled only through the main load bering structure (see Fig. 2).

The influence of the inertial amplification can be estimated as follows

$$
\omega_{t, I A}=\sqrt{\frac{k}{m_{c}+4 \cdot m_{a} \cdot \cot ^{2}(\theta)}} .
$$

The inertial amplification uses amplified lateral vibrations of the secondary masses to increase the inertia within the resonator above the actual mass that is added. The amplification factor for a given masses is dependent on the angle which defines the kinematics within the inertial amplification mechanism. 


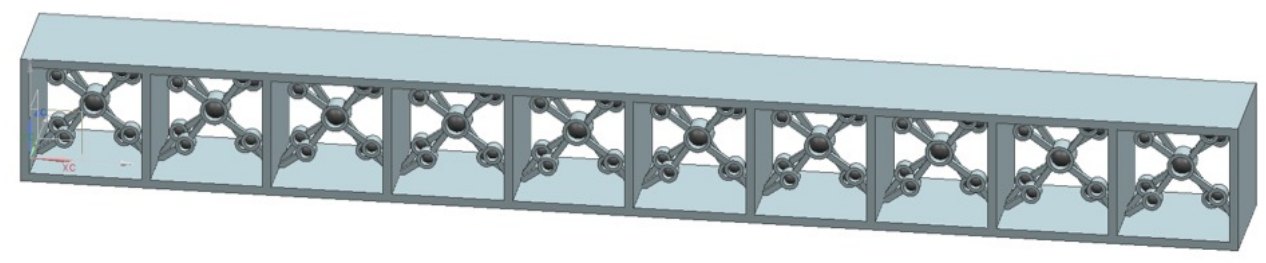

Figure 2. The concept of a load-bearing metamaterial beam.

\subsection{Modelling and Numerical Validation}

The presented load carrying beam with internal resonators has been modelled with a full 3D FE model with volume elements and with a reduced FE model consisting of a full 3D outer structure and the internal resonators consisting of point masses and ideal springs. The simplified model was used to investigate the influence of the single resonators and the inertial amplification on the vibration attenuation.

The results show the relationship between the index of the attenuated eigenmode and the vibration attenuation behavior of such a locally resonant metamaterial. The influence of the index of the targeted eigen mode of the undamped beam can be seen in figure 3. The relative width and also the slope of the edges of the attenuation region increases with the index of the targeted eigen mode $i$.

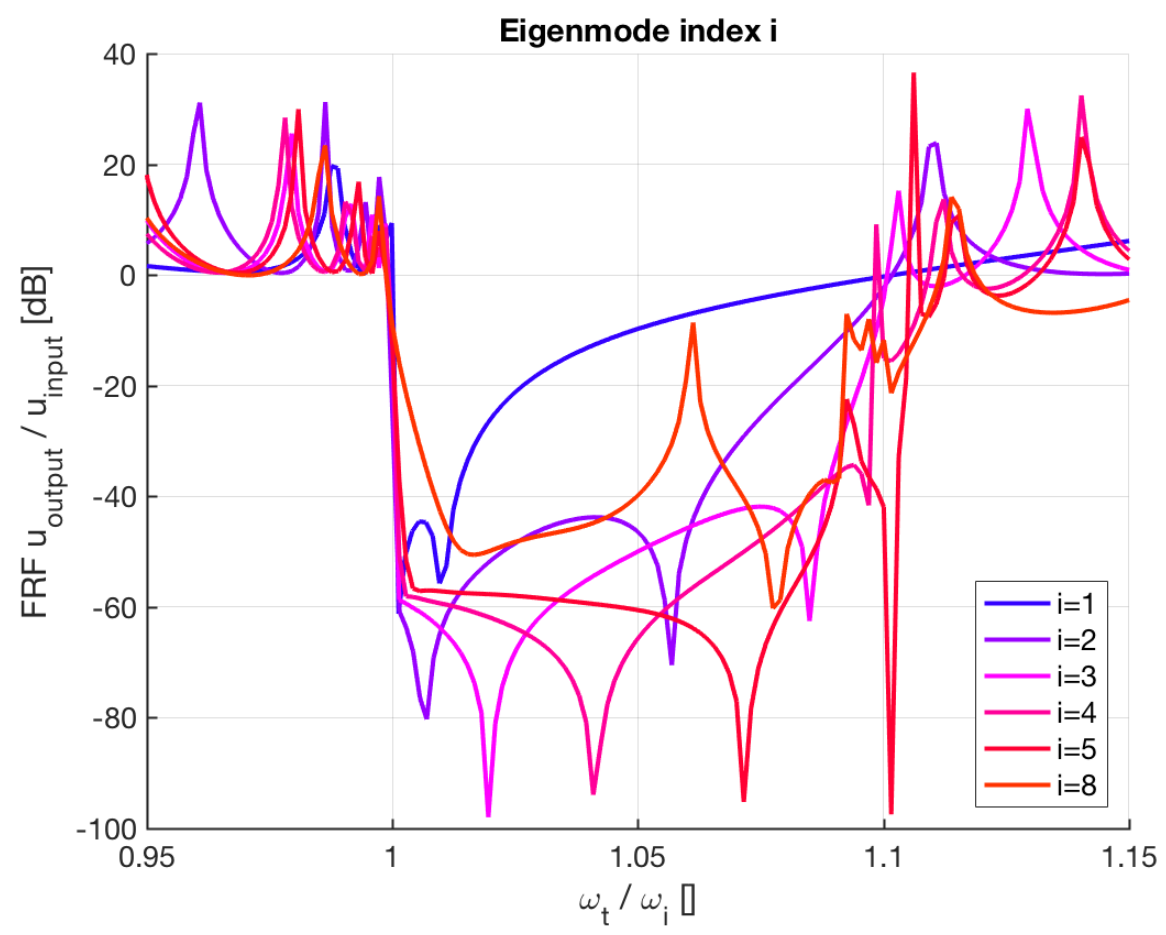

Figure 3. Comparison of the normalized attenuation region shape in the frequency response for different targeted natural frequencies (index $i$ ) of the undamped reference beam for a unit cell with single resonators.

While using a single resonator leads to a limited design space as only the stiffness and the mass of the resonator can be adjusted to achieve low frequency damping. The stiffness of the resonator has a lower limit set by manufacturing and strength considerations, whereas the amount of added mass is limited by the lightweight approach. As a result, the inertial amplification is introduced to extend the design freedom through the design of the kinematics within the inertial amplification mechanism. 
The influence of the angle $\alpha$ defining the kinematics of the inertial amplification mechanism within the resonators is shown in figure 4. A decrease of the angle $\alpha$ results in an increase of the inertial amplification within the resonator, as the tuning frequency of the resonator drops below the reference value set by the single resonator with all mass concentrated in the middle as seen in figure 4 top. The configuration chosen in this case is described by a mass ratio between the primary mass in the center $m_{c}$ and the secondary masses $m_{a}$ is $\frac{m_{c}}{m_{a}}=10$. In figure 4 bottom, it can be seen, that the inertial amplification not only decreases the tuning frequency $\omega_{t}$, but also the width of the attenuation region $\Delta \omega$.
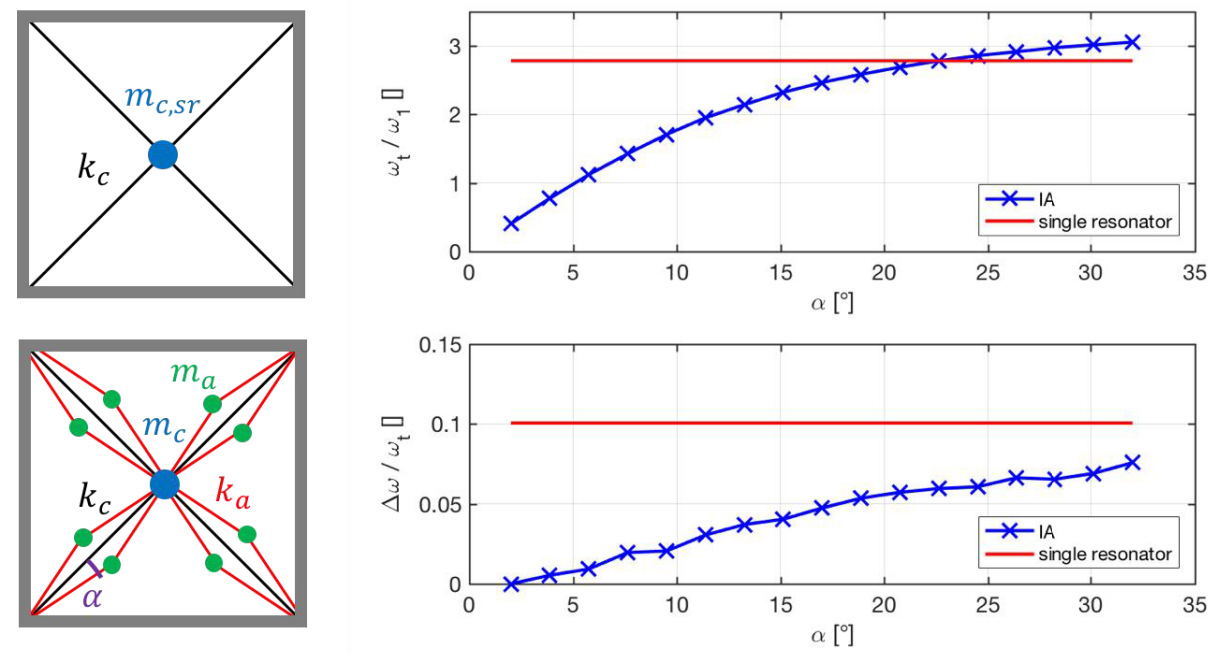

Figure 4. Influence of the inertial amplification on the tuning frequency and the band gap width of the resonator for a given amount of added mass $\mu=\frac{S \cdot\left(m_{c, s r}\right)}{m_{\text {structure }}}=\frac{S \cdot\left(m_{c}+8 \cdot m_{a}\right)}{m_{s} \text { tructure }}$. The single resonator (top left) is the baseline, while the inertial amplification (bottom left) reduces the tuning frequency $\omega_{t}$ depending on the angle $\alpha$.

For the experimental validation, a manufacturable configuration of the inertial amplification mechanism was chosen. The design and the experimental results are presented in the next section. The inertial amplification mechanism in the following investigations has a fixed angle of $\alpha=10 \mathrm{deg}$.

\subsection{Experimental Validation}

The beam has been manufactured by SLS (selective laser sintering), and the masses are added a posteriori in form of tungsten or steel rods or spheres. In this design, the masses and the ligaments in the resonators do not only resonate, but they also add static stiffness to the beam. The 3D printed prototype can be seen in figure 5 .

The 3D-prototype is printed in a way that the masses can be removed and also replaced to test different configurations. As a result, tests with different added mass ratios can be performed. Additionally, by either adding all masses within the unit cell or only the central masses, the frequency response of the beam can be tested with either only simple resonators within the unit cell, or with inertial amplification within the resonators.

\section{RESULTS AND DISCUSSION}

In figure 6 a comparison between the numerical predictions with a finite element model and the experimental results is displayed.

With this model, the influence of the tuning frequency $\omega_{t}$ and the added mass ratio $\mu$ was investigated and a comparison between the analytical predictions, the numerical, and the experimental results are presented in figure 7. The idealized analytical predictions overestimate the width of the attenuation region, while the numerical calculations yield a more moderate value. The fact that the analytical formula overestimates the bandgap is expected, since the motion of the structurally integrated resonators is more complex than the idealized scenario of figure 1 and other scenarios studied by Sugino et al. ${ }^{1}$ (therefore the analytical formula provides an upper 

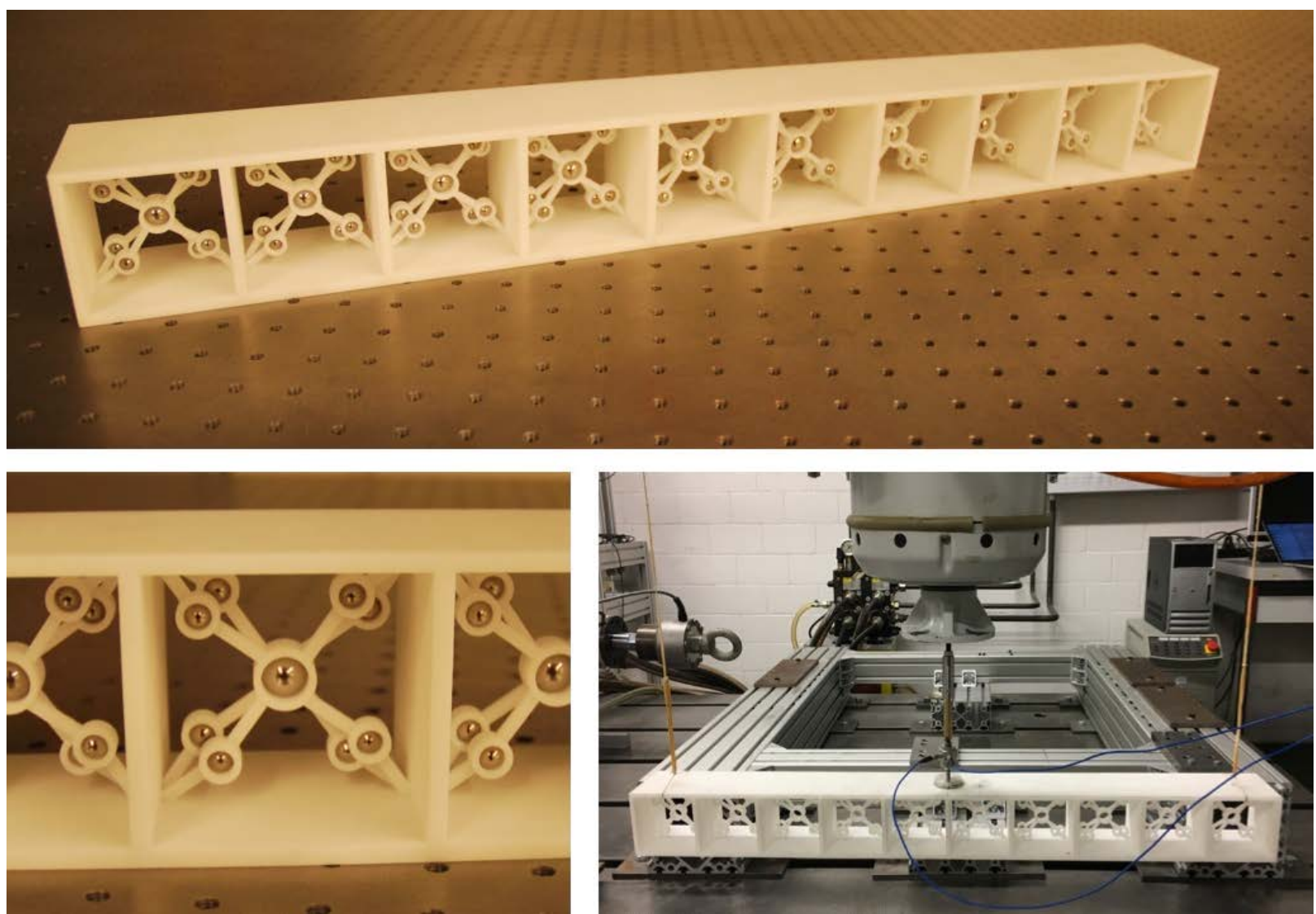

Figure 5. The 3D-printed prototype made from PA12 containing steel spheres uses specific kinematics to create inertial amplification in the resonators for the secondary masses. The experimental setup tests the prototype in a cantilever setting with a excitation near the base and the measurement of the response at the tip.

bound). The experimental results are between these two values. However, the experimental and numerical results match well. It has to be noted, that the definition of the width of the band gap and the band gap itself becomes blurred when looking at the experimental and the numerical results. The resonance of the internal resonators is visible, but the attenuation region is shallow and its edges are not clearly defined. The measure taken to assess the width of the bandgap was chosen as the distance between the midpoints of the slopes at the left and the right border of the band gap. Where the slopes were defined as the FRF between the intersection of the FRF with and without resonators and the first local minimum within the band gap beside it.

This work shows that the inertial amplification can be used to reduce the tuning frequency for a given added mass ratio and a given stiffness of the resonators, however, this comes at the cost of reducing the width of the attenuation region.

\section{CONCLUSIONS AND OUTLOOK}

This work has shown, that it is possible to additively manufacture load bearing structures with integrated resonators, and obtain additional degrees of freedom to shape its dynamic response. Compared to non-load bearing structures with attached resonators for vibration attenuation it is possible to integrate the resonators into a stiff load bearing structure and also design the resonators in such a way, that they are multifunctional and not only act as resonators, but also contribute to the load carrying capability and increase the shear stiffness of the structure.

The introduction of inertial amplification within the resonators increased the weight efficiency concerning achieving low tuning frequencies. However, this resulted in narrower band gaps. It was also not possible to 

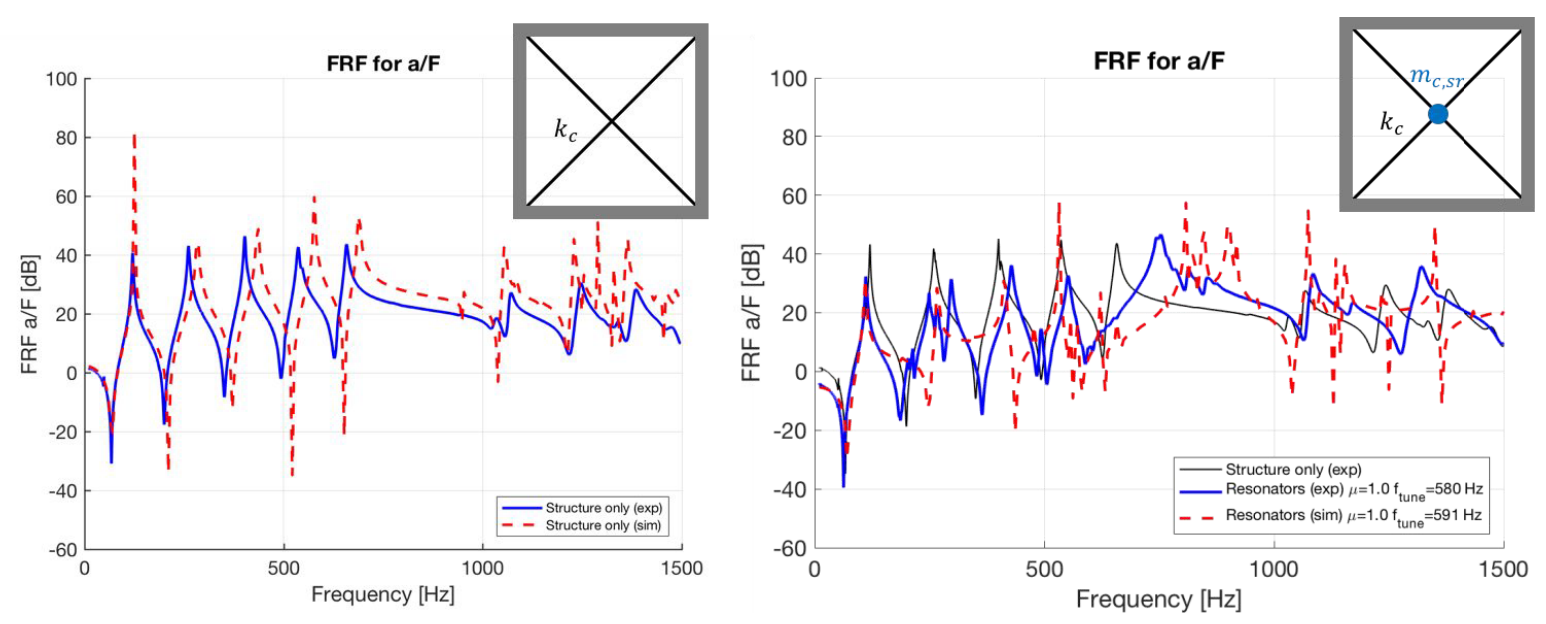

Figure 6. The experimental validation with the 3D printed beam without resonators and with resonators .
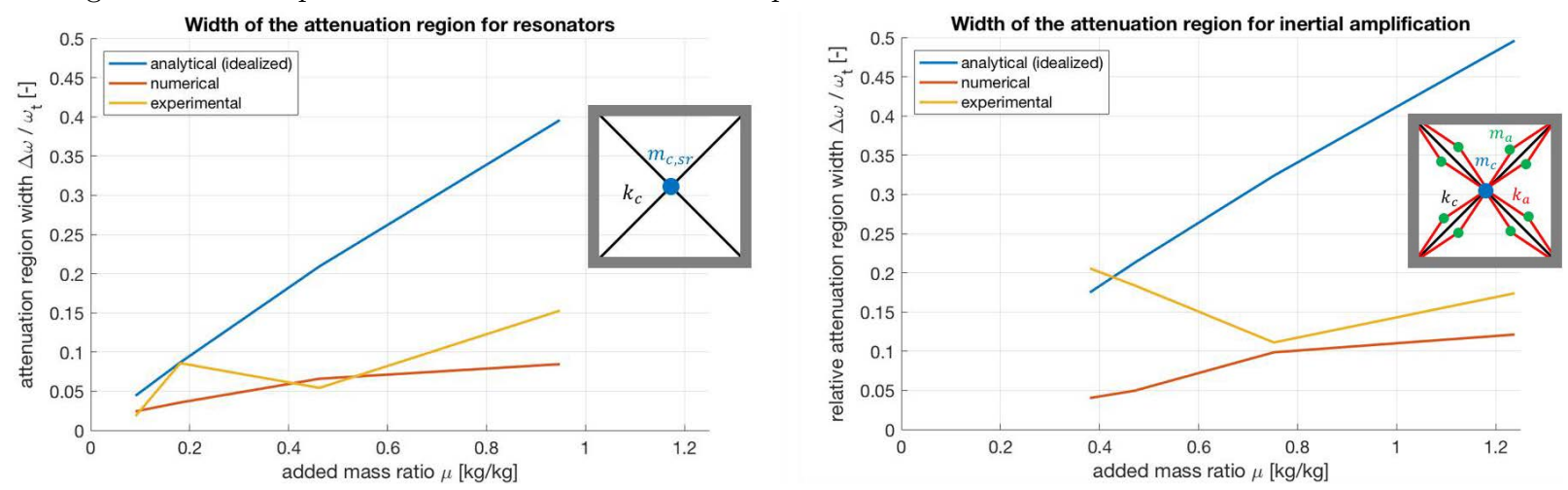

Figure 7. Comparison of the bandgap width for different added mass ratios $\mu$ for the single resonator (left) and for the inertial amplification (right).

completely decouple the width of the attenuation region from the added mass ratio, which was identified as a a critical parameter in designing load bearing structures based on metamaterial used for vibration attenuation.

Further work has to be focused on developing design strategies using single material 3D printing without any further assembly in order to facilitate the manufacturing process. This would in turn create a concept which could be broadly applied in load bearing structure where it may be necessary to attenuate critical eigenfrequencies or decouple harmonic disturbances from the rest of the structure. This metamaterial approach could then be applied as, for example, a core material in sandwich structures. Another future work of interest is to extend the analytical bandgap formulation to complex and structurally-integrated scenarios such as the one explored in this work.

\section{ACKNOWLEDGMENTS}

This work was supported by the Air Force Office of Scientific Research grant FA9550-15-1-0397 "Integrated multi-field resonant metamaterials for extreme, low frequency damping."

\section{REFERENCES}

[1] Sugino, C., Leadenham, S., Ruzzene, M., and Erturk, A., "On the mechanism of bandgap formation in locally resonant finite elastic metamaterials," Journal of Applied Physics 120, 134501 (2016).

[2] Sugino, C., Xia, Y., Leadenham, S., Ruzzene, M., and Erturk, A., "A general theory of bandgap estimation in locally resonant metastructures," Journal of Sound and Vibration submitted (Dec 2016). 


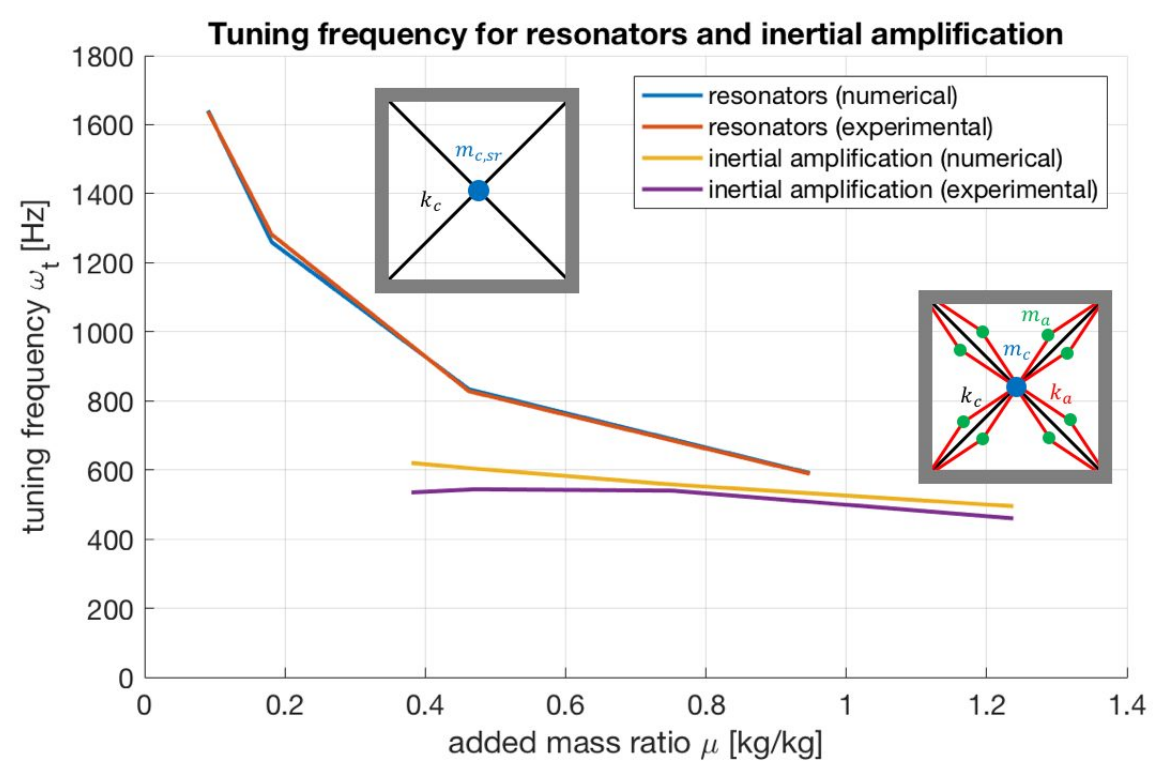

Figure 8. Comparison of the tuning frequency $\omega_{t}$ for different added mass ratios $\mu$ while the stiffness of the resonators is kept constant.

[3] Yilmaz, C., Hulbert, G., and Kikuchi, N., "Phononic band gaps induced by inertial amplification in periodic media," Physical Review B 76(054309) (2007).

[4] Yilmaz, C. and Hulbert, G., "Theory of phononic gaps induced by inertial amplification in finite structures," Physics Letters A 374, 3576-3584 (2010).

[5] Taniker, S. and Yilmaz, C., "Phononic gaps induced by inertial amplification in bcc and fcc lattices," Physics Letters A 377, 1930-1936 (2013). 